\title{
Two-step recanalization technique for a pancreatic duct stricture complicated by massive pancreatic stones
}
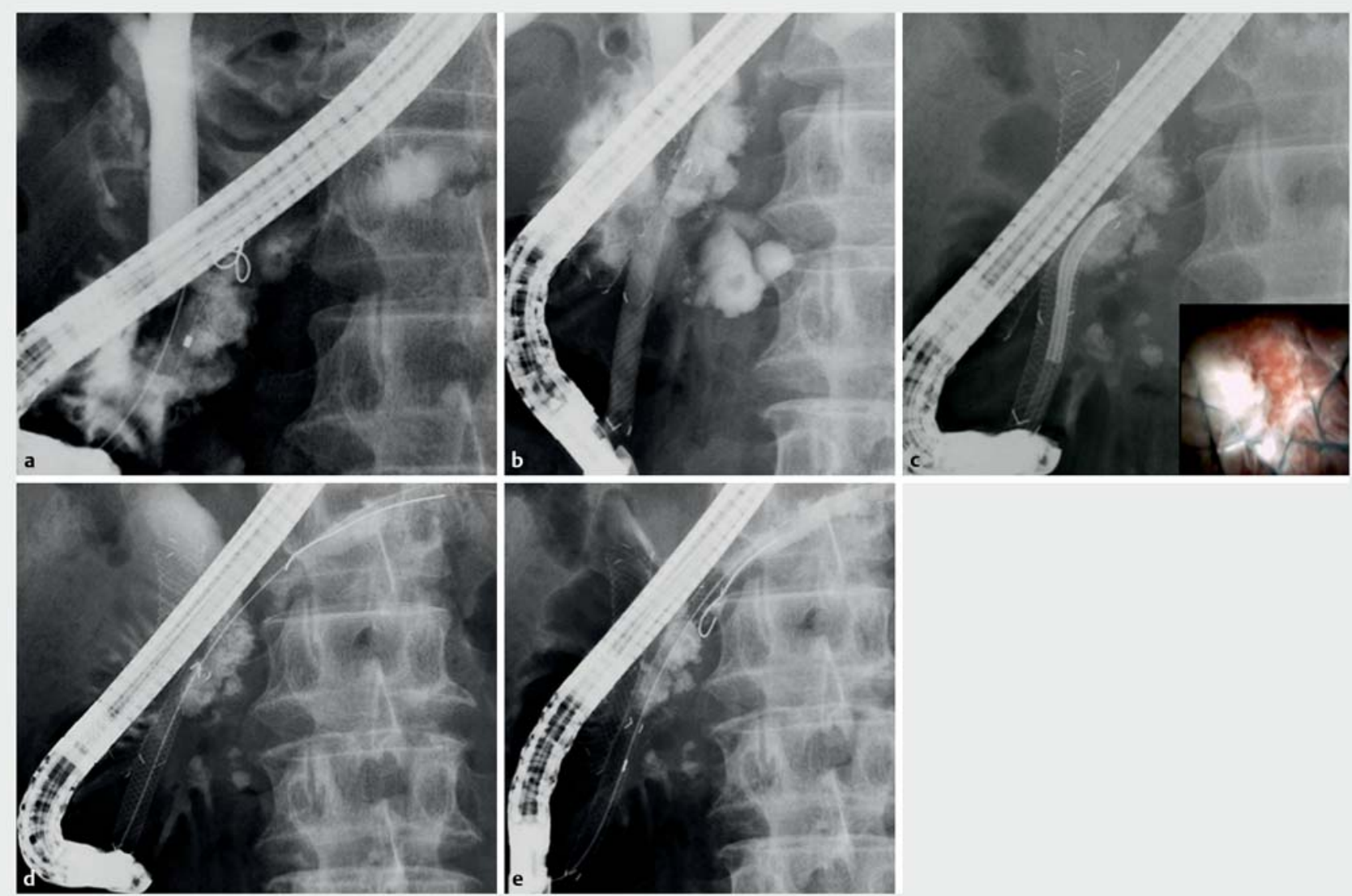

- Fig. 1 Radiographic images during endoscopic retrograde cholangiopancreatography showing: a massive stones in the pancreatic duct that prevented a guidewire being advanced to the pancreatic tail; $\mathbf{b}$ a metal stent deployed over a stricture in the pancreatic duct; $\mathbf{c}$ electrohydraulic lithotripsy being performed through the metal stent, which achieved partial stone fragmentation; $\mathbf{d}$ a guidewire reaching the pancreatic tail, having been passed through the pancreatic duct stones; e a covered metal stent deployed across the pancreatic duct stones.

Electrohydraulic lithotripsy (EHL) under endoscopic retrograde cholangiopancreatography (ERCP) guidance has been established as a treatment option for pancreatic duct stones (PDSs) [1-3]. However, a complicated pancreatic duct stricture or PDS located in the pancreatic head can cause difficulties with pancreatoscope insertion and PDS visualization, thereby rendering $\mathrm{EHL}$ challenging. Here, we describe technical tips for the two-step recanalization of a pancreatic duct stricture complicated by massive PDSs.
A 51-year-old woman with chronic pancreatitis was admitted because of abdominal pain. Computed tomography (CT) scanning revealed massive PDSs in the pancreatic head with pancreatic duct dilatation. Therefore, endoscopic treatment was attempted under ERCP guidance. An ERCP catheter (MTW Endoskopie, Düsseldorf, Germany) was inserted into the pancreatic duct; this was followed by the injection of contrast medium, which confirmed PDSs impacted in the pancreatic head.
We attempted to insert a 0.025 -inch guidewire (VisiGlide; Olympus Medical Systems, Tokyo, Japan) into the pancreatic duct but it could not be advanced to the pancreatic tail across the massive PDSs ( $\vee$ Fig. 1 a). In addition, EHL was not an option because the pancreatoscope (SPY-DS; Boston Scientific, Natick, Massachusetts, USA) could not be inserted owing to a pancreatic duct stricture. Therefore, a $6-\mathrm{mm} \times 6-\mathrm{cm}$, Niti-S Biliary S-Type Long Suture metal stent (TaeWoong Medical, Seoul, South Korea) 


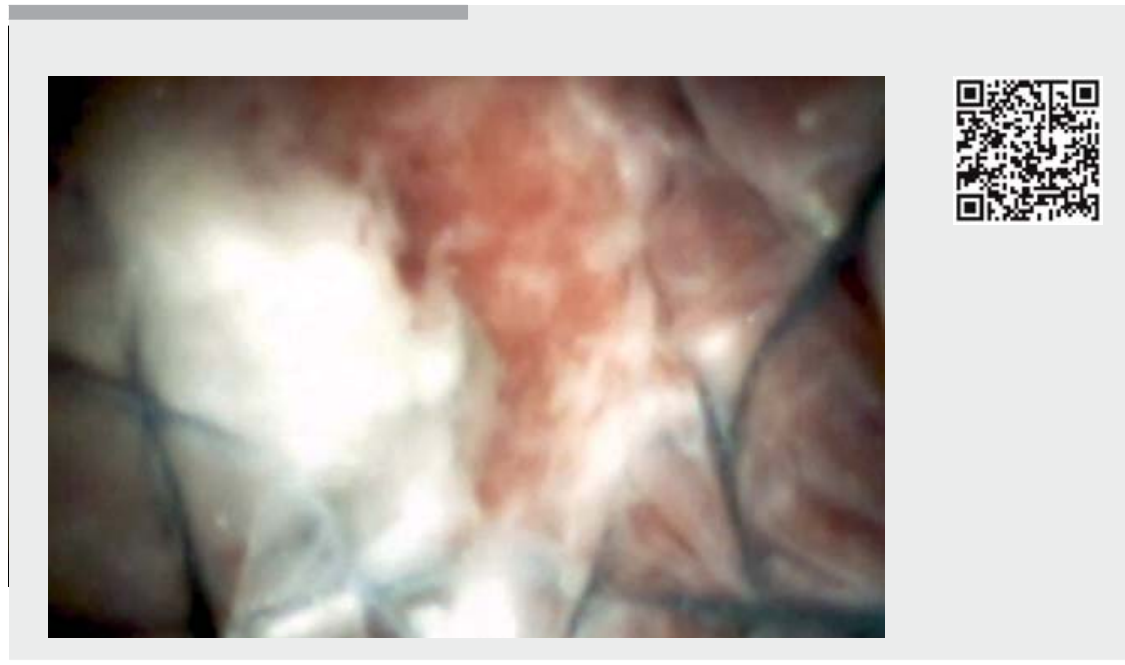

$\checkmark$ Video 1 A pancreatoscope is inserted through a metal stent, which allows visualization of the pancreatic duct stones. Electrohydraulic lithotripsy achieves partial fragmentation of the stones, a guidewire is then inserted into the duct and across the pancreatic stones to the pancreatic tail. Finally, a metal stent is deployed.

was first deployed ( $\bullet$ Fig. $\mathbf{1} \mathbf{b}$ ) and fully expanded.

The pancreatoscope was then inserted into the pancreatic duct through the stent, which allowed visualization of the PDSs ( $\triangleright$ Fig. 1 c; $>$ Video 1 ). EHL was attempted and the stone was partially fragmented, which allowed the guidewire to be advanced to the pancreatic tail ( $\triangleright$ Fig. $1 \mathbf{d}$ ). Finally, a metal stent was deployed from the pancreatic tail to the duodenum across the fragmented PDSs without any adverse events ( $\bullet$ Fig. $\mathbf{1}$ e). Following this procedure, all of the patient's symptoms resolved.

This technique offers a clinical benefit for patients with symptomatic chronic pancreatitis complicated by massive PDSs and stricture in the pancreatic head.

Endoscopy_UCTN_Code_TTT_1AR_2AI
Competing interests

None

The authors

Takeshi Ogura, Atsushi Okuda, Miyuki Imanishi, Rieko Kamiyama, Kazuhide Higuchi 2nd Department of Internal Medicine, Osaka Medical College, Osaka, Japan

\section{Corresponding author}

\section{Takeshi Ogura, MD}

2nd Department of Internal Medicine, Osaka Medical College, 2-7 Daigakuchou,

Takatsukishi, Osaka 569-8686, Japan

Fax: +81-72-6846532

oguratakeshi0411@yahoo.co.jp

[1] Devière J. Pancreatic stents. Gastrointest Endosc Clin N Am 2011; 21: 499 - 510

[2] DiMagno EP, DiMagno M]. Chronic pancreatitis: Landmark papers, management decisions, and future. Pancreas 2016; 45: 641 650

[3] Yan LH, Li L, Chen JS. Chronic pancreatitis. Curr Opin Gastroenterol 2017; 33: 396-403

\section{Bibliography}

DOI https://doi.org/10.1055/a-0631-8382

Published online: 19.6.2018

Endoscopy 2018; 50: E246-E247

(c) Georg Thieme Verlag KG

Stuttgart · New York

ISSN 0013-726X

\section{ENDOSCOPY E-VIDEOS}

https://eref.thieme.de/e-videos 